Pacific Journal of Mathematic 


\section{THE PRESERVERS OF ANY ORTHOGONAL GROUP}

\section{E. P. Botta And Stephen Pierce}

Let $L$ be an invertible linear map on the space $M(n, k)$ of $n$-square matrices over a field $k$ of characteristic not 2 . In this paper we classify all such $L$ which preserve a particular orthogonal group of a nonsingular symmetric bilinear form. We use some elementary facts about algebraic groups and an idea of Dieudonné's. There is some indication that our use of an algebraic geometric setting is the proper one for many problems of this type.

There are a considerable number of results which may be paraphrased as follows: "let $L: M(n, k) \rightarrow M(n, k)$ be a linear transformation that preserves some property related to matrix multiplication. Then $L$ is almost an inner automorphism of $M(n, k)$." While the statement of these results exhibits a large degree of similarity, an examination of the proofs reveals almost no similarity. The property in question could be determinant, nonsingularity, or orthogonality.

For examples of such results, see $[4,5,6,7,8,10]$. In particular, an excellent survey is in [6].

We remark that we must assume $L$ is invertible, since Wei's results [10] show that if singular maps are allowed, then pathological cases can occur.

1. Notation. Let $K$ be an algebraically closed field of characteristic not 2. If $n$ is a positive integer, $K^{n}$ will be the vector space of $n$-tuples of elements of $K, M(n, k)$ the algebra of $n$-square matrices over $K, \operatorname{GL}(n, K)$ the group of matrices in $M(n, K)$ with nonzero determinant, and $K\left[x_{1}, \cdots, x_{n}\right]$ the algebra of polynomials in $n$ variables with coefficients in $K$.

2. Algebraic groups. A subset $V$ of $K^{n}$ is called an algebraic set if there exists an ideal $j(V) \subseteq K\left[x_{1} \cdots x_{n}\right]$ such that

$$
V=\left\{p \in K^{n}: f(p)=0 \text { for all } f \in j(V)\right\} .
$$

The ideal $j(V)$ is called the ideal of $V$. If $I$ is an ideal in $K\left[x_{1}, \cdots, x_{n}\right]$ then the ideal $\operatorname{rad}(I)=\left\{f \in K\left[x_{1}, \cdots, x_{n}\right]: f^{r} \in I\right.$ for some non-negative integer $r$ is called the radical of $I$. We say $V$ is an irreducible algebraic set if $\operatorname{rad} j(G)=j(G)$. If $p \in V$ then $M_{p}=\left\{f \in K\left[x_{1} \cdots x_{n}\right]: f(p)=0\right\}$ is a maximal ideal and if $p=\left(a_{1}, \cdots, a_{n}\right)$ we have $M_{p}=$ 
$\left\langle x_{1}-a_{1}, \cdots, x_{n}-a_{n}\right\rangle$. Conversely, given a maximal ideal $M$ in $K\left[x_{1} \cdots x_{n}\right]$, we have that there exists a unique point $p \in K^{n}$ such that $\boldsymbol{M}=M_{p}$.

If $V \subseteq K^{n}$ and $W \subseteq K^{m}$ are algebraic sets then a function $F: V \rightarrow W$ is called a polynomial function if there exist $F_{1}, \cdots, F_{m} \in$ $K\left[x_{1}, \cdots, x_{n}\right]$ such that for all $v \in V F(v)=\left(F_{1}(v), \cdots, F_{m}(v)\right)$. The algebra of all polynomial functions $f: V \rightarrow K$ is called the co-ordinate ring of $V$ and is denoted by $K[V]$. It is easy to see that

$$
K[V] \cong K\left[x_{1}, \cdots, x_{n}\right] / j(V)
$$

and the isomorphism is given by $f \leftrightarrow F+j(V)$ where $f \in K[V]$ and $F \in K\left[x_{1} \cdots x_{n}\right]$ is such that $f(v)=F(v)$ for all $v \in V$.

If $V \subseteq K^{n}$ and $W \subseteq K^{m}$ are algebraic sets and $F: V \rightarrow W$ a polynomial function define $\bar{F}: K[W] \rightarrow K[V]$ by $\bar{F}(f)=f \circ F$. It is easily verified that $\bar{F}$ is an algebra homomorphism. If $\phi: K[W] \rightarrow K[V]$ is an algebra homomorphism define $\hat{\phi}: V \rightarrow W$ as follows: If $p \in V$ let $M_{p}$ be the maximal ideal associated with $M_{p}$ and $\mathcal{M}_{p}$ the image of $M_{p}$ in $K[V]$. Then $\mathcal{M}_{p}$ is a maximal ideal in $K[V]$ and $\phi^{-1}\left(\mathcal{M}_{p}\right)$ is a maximal ideal in $K[W]$ so $\phi^{-1}\left(\mathcal{M}_{p}\right)=\mathcal{M}_{q}$ for some $q \in \mathcal{W}$. Set $\phi(p)=q$. One verifies that $\hat{\phi}$ is a polynomial function. Further $\hat{\phi}=\phi$ and $\hat{\bar{F}}=F$.

If $G \leqq G L(n, K)$ we shall say $G$ is an algebraic group if $G$ is a subgroup of $\operatorname{GL}(n, K)$ and $G$ is an algebraic set. If $g \in G$ and $x \in M(n, K)$ we say that $x$ is tangent to $G$ at $g$ if

$$
\left.\frac{d}{d t} f(g+t x)\right|_{t=0}=0 \quad \text { for all } f \in j(G) .
$$

The set of all tangents to $G$ at $g$ is called the tangent space to $G$ at $g$ and is denoted by $T_{g}(G)$ or simply $T_{g}$ if $G$ is understood. If 1 is the identity matrix we write $T_{1}(G)=\Omega$ and call $\Omega$ the tangent space to $G$. As is well known $\Omega$ is a Lie algebra and $T_{g}=g \Omega$.

3. Linear mappings on algebraic groups. Let $G$ be an algebraic group and suppose $L: M(n, K) \rightarrow M(n, K)$ is a linear mapping with the properties that $L$ is nonsingular, $L(1)=1$ and $L(G) \leqq G$.

Proposition 3.1. If $g \in G$ then $L\left(T_{g}\right)=T_{L(g)}$. Hence $L(g \Omega)=$ $L(g) \Omega$.

Proof. Clearly $L$ is a polynomial function and since $L(G) \leqq G$ clearly $F \in j(G)$ implies $F \circ L \in j(G)$. Now suppose $x \in T_{g}$. Then

$$
\left.\frac{d}{d t} f(g+t x)\right|_{t=0}=0 \quad \text { for all } f \in j(G) .
$$


Now, if $F \in j(G)$ we have

$$
\begin{aligned}
\left.\frac{d}{d t} F((L(g)+t L(x)))\right|_{t=0} & =\left.\frac{d}{d t}(F(L(g+t x)))\right|_{t=0} \\
& =\left.\frac{d}{d t}(F \circ L(g+t x))\right|_{t=0} \\
& =0 \text { since } F \circ L \in j(G), x \in T_{g} .
\end{aligned}
$$

Hence $L(x) \in T_{L(g)}$. Since $g$ and $L(g)$ are nonsingular, we may conclude that $\operatorname{dim}(\Omega)=\operatorname{dim}(g \Omega)=\operatorname{dim}(L(g) \Omega)$; and then since $L$ is nonsingular, we may conclude that $L(g \Omega)=L(g) \Omega$.

Proposition 3.2. If $g \in G$ then $L(g \Omega \cap \Omega)=L(g) \Omega \cap \Omega$.

Proof. Clearly $L(g \Omega \cap \Omega) \subseteq L(g \Omega) \cap L(\Omega)=$ (by 3.1) $L(g) \Omega \cap \Omega$. Conversely, suppose $u \in L(g) \Omega \cap \Omega$. Then $L^{-1}(u) \in L^{-1}(L(g) \Omega \cap \Omega)$ = (by 3.1) $L^{-1}(L(g \Omega) \cap L(\Omega))=($ since $L$ is a bijection) $g \Omega \cap \Omega$.

DEFINITION 3.3. Let $r$ be a nonnegative integer and define

$$
G(r)=\{g \in G: \operatorname{dim}(g \Omega \cap \Omega)=r\} .
$$

Proposition 3.4. $L(G(r)) \subseteq G(r)$.

Proof. If $g \in G(r), \quad \operatorname{dim}(g \Omega \cap \Omega)=r$; however we have $L(g \Omega \cap \Omega)=L(g) \Omega \cap \Omega$ and since $L$ is nonsingular it follows that $r=\operatorname{dim}(g \Omega \cap \Omega)=\operatorname{dim} L(g \Omega \cap \Omega)=\operatorname{dim}(L(g) \Omega \cap \Omega)$.

\section{Extensions of fields and related algebraic groups.}

Proposition 4.1. Let $k \subseteq K$ be fields. Suppose $G \leqq G L(n, k)$ is an algebraic group and $L: M(n, k) \rightarrow M(n, k)$ a nonsingular linear mapping with the property that $L(G) \subseteq G$. Let $G^{K}$ be the smallest algebraic subgroup of $\mathrm{GL}(n, K)$ containing $G$. Then

(a) $G^{K} \cap M(n, k)=G$

(b) there exists a nonsingular linear mapping $L^{K}: M(n, K) \rightarrow M(n, K)$ with the properties that $L^{K}\left(G^{K}\right) \subseteq G^{K}$ and $L^{K} \mid M(n, k)=L$.

Proof. Part (a) follows from Chevalley [2], Ch. II, Théorèm 3. To establish part (b) choose a basis for $M(n, K)$ out of $M(n, k)$ and let $L^{K}$ be the linear mapping of $M(n, K)$ defined by the matrix of $L$ with respect to 
this basis. Since the determinants of $L$ and $L^{K}$ are the same $L^{K}$ is nonsingular and clearly $L^{K} \mid M(n, k)=L$. It only remains to show that $L^{K}\left(G^{K}\right) \subseteq G^{K}$. It is known [Chevalley (loc. cit.)] that the ideal associated with $G^{K}$ is the ideal formed by all linear combinations of elements of $j(G)$ with coefficients from $K$. Now suppose $f$ belongs to $j\left(G^{K}\right)$ and $g$ belongs to $G^{K}$. Then $f=\sum_{i=1}^{m} a_{i} f_{i}$ for some $a_{i}$ in $K$ and $f_{i}$ in $j(G)$; hence

$$
\begin{aligned}
f\left(L^{K}(g)\right) & =\sum_{i=1}^{m} a_{i} f_{i}\left(L^{K}(g)\right) \\
& =\sum_{i=1}^{m} a_{i} \bar{L}^{K}\left(f_{i}\right)(g) \\
& =\sum_{i=1}^{m} a_{i} \bar{L}\left(f_{i}\right)(g)=0
\end{aligned}
$$

since $L\left(f_{i}\right) \in j(G) \subseteq j\left(G^{K}\right)$ and $\quad \bar{L}^{K}\left(f_{i}\right)=L\left(f_{i}\right)$ since $f_{i} \in j(G)$. Therefore $L^{K}(g)$ belongs to $G^{K}$.

\section{The orthogonal groups}

DeFinition 5.1. Let $A$ be an invertible symmetric matrix in $M(n, k)$. Then $G$ is an orthogonal group if $G=\left\{X \mid X^{\prime} A X=A\right\}$.

Proposition 5.2. Let $L: M(n, k) \rightarrow M(n, k)$ be linear and let $L(G)=G$ where $G$ is an orthogonal group. If $K$ is the algebraic closure of $k$, then $L^{K}\left(G^{K}\right)=G^{K}$.

Proof. Clear from Proposition 4.1.

Definition 5.3. Let $K$ be algebraically closed and $G$ an orthogonal group in $M(n, K)$. Let $O(n, K)=\left\{X \mid X^{T} X=I_{n}\right\}$. If $L(G)=G$, define a linear map $\hat{L}: O(n, K) \rightarrow O(n, K)$ by the diagram

$$
O(n, K) \stackrel{\bar{B}}{\longrightarrow} G \stackrel{L}{\longrightarrow} G \stackrel{\tilde{B}^{-1}}{\longrightarrow} O(n, K)
$$

In the diagram, $\tilde{B}$ is conjugation by a matrix $B$ such that $B G B^{-1}=$ $O(n, K)$. Such a matrix $B$ exists because $K$ is algebraically closed, and we may write $A=B^{T} B$.

Proposition 5.4. $L$ is a G-conjugation if and only if $\hat{L}$ is an $O(n, K)$-conjugation. If $\hat{L}$ is the transpose map, then $L$ is the transpose map followed by similarity by $A$. 
Proof. The first part is clear from the diagram. In the second case, let $A$ be the matrix of Definition 5.1. Write $A=B{ }^{T} B$. Then $B$ is the matrix we need. Let $U \in G$. If $\hat{L}$ is the transpose map,

$$
L(U)=\tilde{B} \hat{L} \tilde{B}^{-1}(U)=B^{-1} B^{-1^{T}} U^{T} B^{T} B=A^{-1} U^{T} A .
$$

Clearly, since $U \in G, A^{-1} U^{T} A \in G$.

6. The main theorem. Let $K$ be any field of characteristic $\neq 2$, and let $G$ be any orthogonal group in $M(n, K)$. If $L$ is an invertible linear map on $M(n, K)$ such that $L\left(I_{n}\right)=I_{n}$, and $L(G)=G$, then $L$ is conjugation by a matrix in $M(n, \tilde{K})$ where $\tilde{K}$ is the algebraic closure of $K$, or $L$ is such a conjugation followed by the transpose map.

REMARK 6.1. By $\S 5$, we will assume henceforth that $K$ is algebraically closed and that $G=O(n, K)$.

REMARK 6.2. The assumption $L\left(I_{n}\right)=I_{n}$ is just a normalization of the problem. The Main Theorem can be modified slightly and then $L\left(I_{n}\right)=I_{n}$ can be dropped.

7. The characterization of symmetries. Recall that a symmetry in $G$ is an involution sending a nonisotropic vector $v$ to $-v$ and fixing pointwise the orthogonal compliment of $v$.

We wish to characterize symmetries and their negatives in a manner which will be invariant under $L$. Let $\Omega$ be the tangent space of $G$; in this case, since $G=O(n, K), \Omega$ is all skew symmetric matrices. Let $V=K^{n}$ be the underlying vector space.

Proposition 7.1. Let $A \in G, A \neq \pm I_{n} n \geqq 3$. Then

$$
\operatorname{dim}(A \Omega \cap \Omega) \leqq(n-1)(n-2) / 2
$$

with equality if and only if $A= \pm$ a symmetry.

REMARK 7.2. In view of 3.4 and $7 . \dot{1}$, any $L$ satisfying $L(G) \subseteq G$ also sends symmetries to \pm symmetries. This fact enables us to use the fundamental theorem of projective geometry in proving the main theorem for $n \geqq 3$. The main theorem has to be proved separately for $n=2$. Our proof of 7.1 will be by induction on $n$.

Now suppose equality holds in (1). We consider three cases.

Case 1. $A$ has a non-isotropic eigenvector $v$. Let $A v=\lambda v$. Clearly $\lambda= \pm 1$. We are trying to show that $\pm A$ is a symmetry, so assume $\lambda=-1$. Now $v$ is nonisotropic; thus $A$ is $G$-conjugate to a matrix $B=-1+B_{1}$. If $B_{1}=I_{n-1}$, we are done; if not, we show $B_{1}$ is the 
negative of a symmetry. Let $\Omega_{1}$ be the $(n-1) \times(n-1)$ skew-symmetric matrices. By induction

$$
\operatorname{dim}\left(B_{1} \Omega_{1} \cup \Omega_{1}\right) \leqq(n-2)(n-3) / 2 .
$$

Put

$$
W=\left[\begin{array}{c|c}
0 & x \\
\hline-x^{\prime} & W_{1}
\end{array}\right] \in \Omega .
$$

Then $B W \in \Omega$ if and only if $B_{1} W_{1} \in \Omega_{1}$ and $B_{1} x^{\prime}=-x^{\prime}$. The set of all $x^{\prime}$ such that $B_{1} x^{\prime}=-x^{\prime}$ has dimension no larger than $n-2$ and hence

$$
\begin{aligned}
\operatorname{dim}(B \Omega \cap \Omega) & \leqq(n-2)(n-3) / 2+(n-2) \\
& =(n-1)(n-2) / 2
\end{aligned}
$$

with equality holding if and only if $B_{1}$ is - a symmetry. This establishes Case 1.

In the final two cases we assume all eigenvectors of $A$ are isotropic.

Case 2. A has two isotropic eigenvectors $v, w$ which generate a hyperbolic plane. Let $A v=\lambda v, A w=\mu w$, with $(w, w)=(v, v)=0$, and $(w, v)=1$. Thus, $\lambda \mu=1$. Since $G$ acts transitively on hyperbolic planes, $A$ is $G$-conjugate to a matrix $B$ of the form $B=\left[\begin{array}{ll}a & b \\ c & d\end{array}\right]+B_{1}$ with $\left[\begin{array}{ll}a & b \\ c & d\end{array}\right]$ having eigenvalues $\lambda, \mu$.

If $b$ or $c=0, B$ has non-isotropic eigenvectors. Thus, $b c \neq 0$.

Let $W \in \Omega$ and write

$$
W=\left[\begin{array}{cc|c}
0 & w & x \\
-w & 0 & \\
\hline-x^{\prime} & W_{1}
\end{array}\right]
$$

where $x=\left[\begin{array}{l}x_{3} \cdots x_{n} \\ y_{3} \cdots y_{n}\end{array}\right]$. If $B W \in \Omega$, we must have $w=0$ and $B_{1} W_{1}$ skew. The set of all $W_{1}$ for which this is true has dimension $<(n-3)(n-4) / 2$ because $B_{1}$ has no non-isotropic eigenvectors. Since $b c \neq 0$, there is, for each $i=3, \cdots, n$, a dependence relation between $y_{i}$ and all $x_{i}$. It follows that 


$$
\begin{aligned}
\operatorname{dim}(B \Omega \cap \Omega) & <\frac{(n-3)(n-4)}{2}+n-2 \\
& =\frac{(n-1)(n-2)+6-2 n}{2} \\
& \leqq(n-1)(n-2) / 2
\end{aligned}
$$

if $n \geqq 3$. This finishes Case 2 .

Case 3. All eigenvectors of $A$ are isotropic and any two eigenvectors of $A$ are orthogonal. It follows that the subspace $U$ of $V$ spanned by the eigenvectors of $A$ is totally isotropic and hence its dimension is $\leqq[n / 2]$.

Now if $W$ and $A W \in \Omega$, then $(A W)^{\prime}=-A W$, i.e., $A W-W A^{\prime}=$ 0 . Thus the map $H=I_{n} \otimes A-A \otimes I_{n}$ has $W$ in its null space. Therefore, we must show that the restriction of $H$ to $\wedge^{2} V$ has nullity $<(n-1)(n-2) / 2$. Call this restriction $\hat{H}$. Clearly this nullity is maximized if all eigenvalues of $H$ are the same, say all are 1.

Let $(\lambda-1)^{m_{i}}, i=1, \cdots, r$, be the elementary divisors of $H$. For $v, w \in V$, put $v \circ w=(v \otimes w+w \otimes v) / 2$. The span of all $v \circ w$ is $V^{(2)}$, the symmetric tensors. Clearly $\hat{H} ; \wedge^{2} V \rightarrow V^{(2)}$. If $(\lambda-1)^{m}$ is an elementary divisor of $A$, pick $v_{1}, \cdots, v_{m} \in V$ such that $A v_{1}=v_{1}, A v_{\imath}=$ $v_{\imath}+v_{\imath-1}, \quad i=2, \cdots, m$. By looking at $H\left(v_{1} \wedge v_{i}\right)$, we see that $v_{1} \circ v_{1}, \cdots, v_{1} \circ v_{m-1} \in \operatorname{rng} \hat{H}$. Suppose $(\lambda-1)^{p}$ is another elementary divisor of $A$ and pick $w_{1}, \cdots, w_{p} \in V$ as we did the $v_{l}$. By looking at $\hat{H}\left(v_{1} \wedge w_{1}\right)$ and $\hat{H}\left(v_{1} \wedge w_{1}\right)$, we note that $v_{1} \circ w_{1}$ and $v_{j} \circ w_{1} \in \operatorname{rng} \hat{H}$ for $i=1, \cdots, p-1, j=1, \cdots, m-1$. It follows that the rank of $\hat{H}$ is at least

$$
\begin{aligned}
\sum_{i<j}\left(m_{\imath}-1\right)+\left(m_{j}-2\right)+\sum_{i=1}^{r}\left(m_{i}-1\right) & =(r-1) n-3\left(\begin{array}{l}
r \\
2
\end{array}\right)+n-r \\
& =r(n-(3 r-1) / 2) .
\end{aligned}
$$

A little calculus shows that this value is minimized when $r$ is at its extremes, i.e., when $r=1$ or $[n / 2]$. When $r=1$, we get rank $\hat{H} \geqq$ $n-1$. When $r=[n / 2]$, we get rank $\hat{H}>n^{2} / 8+n / 4$.

If $r=1$, let $A v_{1}=v_{1}, A v_{1}=v_{i-1}, i=2, \cdots, n$. Look at $\hat{H}\left(v_{2} \wedge v_{3}\right)$ $(n \geqq 3)$ to see that $\operatorname{rank} \hat{H} \geqq n$. Then nullity $\hat{H} \leqq\left(n^{2}-n\right) / 2-n<$ $(n-1)(n-2) / 2$.

If $r=[n / 2], \quad$ then nullity $\hat{H} \leqq\left(n^{2}-n\right) / 2-n^{2} / 8-n / 4<$ $(n-1)(n-2) / 2$, unless $n=3$ or 4 . If $n=3$, then $r=1$, and we are done. Let $n=4$. If $(\lambda-1),(\lambda-1)^{3}$ are the elementary divisors of $A$, pick a basis $v, w, x, y$ of $V$ with $A v=v, A w=w, A x=x+w$, 
$A y=y+x$. It is easy to show that $\langle v, w, x\rangle$ is totally isotropic. If $(\lambda-1)^{2},(\lambda-1)^{2}$ are the elementary divisors of $A$, pick a basis $v, w, x, y$ of $V$ with $A v=v, A w=w+v, A x=x, A y=y+x$. Then direct computation shows that $\operatorname{rng} \hat{H}=\langle v \circ v, x \circ x, v \circ x, w \circ x-v \circ y\rangle$. Thus, rank $\hat{H}=4$ and nullity $\hat{H}=2<(4-1)(4-2) / 2$.

We still have to do cases 1 and 2 when $n=3$. Then these two cases coincide. Let $v$ be a nonisotropic eigenvector and assume $A v=v$. If $A \neq \pm I_{3}$ nor \pm a symmetry, there are two possibilities for $\langle v\rangle^{\perp}:\langle v\rangle^{\perp}=$ $\langle u, w\rangle$ and $A u=\lambda u, \quad A w=\lambda^{-1} w, \lambda \neq \lambda^{-1}, \quad$ or $A u= \pm u, \quad A w=$ $\pm w+u$. In the first case, $\hat{H}$ has nullity 0 . In the second case, $V$ is degenerate. This concludes the proof of 7.1.

\section{The fundamental theorem of projective} geometry. We now know that $L$ maps every symmetry to another symmetry or its negative. Thus, since $L$ maps $G$ onto itself, $L$ sets up a $1-1$ correspondence $\phi$ between the nonisotropic lines of $V$. We will extend $\phi$ to all lines of $V$.

Proposition 8.1. There is an extension of $\phi$ to all lines of $V$ such that $\phi$ satisfies the hypothesis of the Fundamental Theorem of Projective Geometry.

Remark. Recall the Fundamental Theorem of Projective Geometry. If $P(V)$ is projective space, and $\phi$ is a bijection of the points of $P(V)$ preserving collinearitry, then $\phi$ is induced by a semilinear map on $V$.

Let $L_{1}, L_{2}, L_{3}$ be nonisotropic lines and let $M_{i}=\phi\left(L_{i}\right)$. We need to show that $\phi$ preserves orthogonality and coplanarity among $L_{1}, L_{2}$, $L_{3}$. If $n \geqq 4$, the proof will then follow from the same procedure as in [3, p. 48]. If $n=3$, we do things a little differently.

Let $\sigma_{i}$ be the symmetry of $L_{i}$ and $\tau_{i}$ the symmetry of $M_{i}, i=1,2,3$.

We now prove the following.

Proposition 8.2. If $\sigma_{1}, \sigma_{2}, \sigma_{3}$ and $L_{1}, L_{2}, L_{3}$ are as above, then

$$
\operatorname{dim}\left(\sigma_{1} \Omega \cap \sigma_{2} \Omega \cap \sigma_{3} \Omega \cap \Omega\right) \leqq(n-2)(n-3) / 2
$$

with equality if and only if $L_{1}, L_{2}$, and $L_{3}$ lie in the same plane.

Proof. Pick an orthonormal basis $v_{1}, \cdots, v_{n}$ such that $\left\langle v_{1}\right\rangle=L_{1}$, and $L_{1}, L_{2} \in\left\langle v_{1}, v_{2}, v_{3}\right\rangle\left(\left\langle L_{1}, L_{2}\right\rangle\right.$ may be degenerate $)$. Represent $\sigma_{1}$ with $A_{1}=-1+I_{n-1}, \sigma_{2}$ with $A_{2}=B+I_{n-3}$ and $\sigma_{3}$ with $A_{3}=\left[\begin{array}{cc}R & S \\ S^{\prime} & U\end{array}\right]$, where $R$ is $3 \times 3$. If 


$$
W=\left[\begin{array}{rr}
W_{1} & W_{2} \\
-W_{2}^{\prime} & W_{4}
\end{array}\right]
$$

is a skew matrix such that $A_{1} W$ and $A_{2} W$ are skew, then an easy computation shows that the first row and column of $W$ are 0 and that $B W_{2}=W_{2}$. Thus, $\operatorname{dim}\left(\Omega \cap \sigma_{1} \Omega \cap \sigma_{2} \Omega\right)=(n-2)(n-3) / 2$ and we are done in one direction.

Now if $A_{3} W$ is also skew, for all $W \in \Omega \cap \sigma_{1} \Omega \cap \sigma_{2} \Omega$, then we obtain $U=I_{n-3}, S=0$ and $R W_{2}=W_{2}$. It follows that $\sigma_{1}, \sigma_{2}, \sigma_{3}$ have a common fixed $(n-2)$-dimensional subspace and thus $L_{1}, L_{2}, L_{3}$ are in the same plane. This proves our result.

We now need to show that $\phi$ preserves orthogonality of nonisotropic lines.

Proposition 8.3. Suppose $\operatorname{dim} V=2$ and $\sigma_{1}, \sigma_{2}$ are distinct symmetries. If for every symmetry $\sigma_{3} \neq \sigma_{1}$ or $\sigma_{2}, \sigma_{1}+\sigma_{2}-\sigma_{3}$ is also a symmetry, then $\sigma_{1}$ and $\sigma_{2}$ reflect in orthogonal lines.

Proof. Let $\sigma_{1} v_{1}=-v_{1}$ and pick $v_{2}$ so that $v_{1} \perp v_{2}$. Let $\sigma_{2} v_{1}=$ $a v_{1}+b v_{2}, \sigma_{2} v_{2}=b v_{1}-a v_{2}$, and $a^{2}+b^{2}=1$. If $\sigma_{3}$ is any other symmetry, we have $\sigma_{3} v_{1}=c v_{1}+d v_{2}, \sigma_{3} v_{2}=d v_{1}-c v_{2}$. Thus, for all $c$ and $d$ such that $c^{2}+d^{2}=1$, we have

$$
\operatorname{det}\left(\begin{array}{cc}
1+a+c & b+d \\
b+d & -1-a-c
\end{array}\right)=-1 .
$$

This can happen only if $a=-1$.

Corollary 8.4. Let $\sigma_{1}, \sigma_{2}$ be two symmetries in an $n$-dimensional nondegenerate space $V, n \geqq 3$. Let $L_{1}, L_{2}$ be the corresponding lines. If, for every symmetry $\sigma_{3} \neq \sigma_{1}$ or $\sigma_{2}$, whose line of reflection is in the plane determined by $L_{1}, L_{2}, \sigma_{1}+\sigma_{2}-\sigma_{3}$ is a symmetry, then $L_{1}$ is orthogonal to $L_{2}$.

The next result finishes Proposition 8.1.

Proposition 8.5. The map $\phi$ preserves orthogonality.

Proof. Let $\sigma_{1}, \sigma_{2}$ be orthogonal symmetries. Let $\sigma_{3}$ be any symmetry whose line is in the plane corresponding to $\sigma_{1}, \sigma_{2}$. Let $\tau_{1}$ be the images. By Proposition 8.2, the lines of $\tau_{1}, \tau_{2}, \tau_{3}$ lie in a plane. We now apply Corollary 8.4 to finish. 
We can now follow the procedures of Dieudonné to extend $\phi$ to all lines of $V$. This extension satisfies the hypotheses of the Fundamental Theorem of Projective Geometry and also preserves orthogonality. To $\phi$, we associate a map $\psi$ in the projective space $P(V)$, and conclude that $\psi$ is induced by a semilinear map $g: V \rightarrow V$. Let $\rho$ be the automorphism of $K$ corresponding to $g$. We can also show that for all $u, v \in V$,

$$
(g(u), g(v))=\lambda(u, v)^{\rho}
$$

where $\lambda$ is independent of $u$ and $v$. It follows that if $A$ is a symmetry, then

$$
L(A)= \pm g A g^{-1}
$$

Now $L$ is linear; thus $\rho=$ identity and $g$ is linear.

Proposition 8.6. The \pm sign is always + , that is, symmetries are mapped to symmetries.

Proof. Let $\sigma_{1}$ be a symmetry. Let $\sigma_{2}, \cdots, \sigma_{n}$ be symmetries such that the $n$ associated lines are pairwise orthogonal. Then $\Sigma \sigma_{i}=$ $(n-2) I_{n}$, and since $L\left(I_{n}\right)=I_{n}$, we are done.

Fix the standard basis $E=\left\{e_{1}, \cdots, e_{n}\right\}$ and represent the symmetries as matrices with respect to $E$. The linear span of the symmetries is the space $S$ of all symmetric matrices in $M(n, K)$. Thus, on $S$, the map $L$ is conjugation. Hence, in Chapter 10, we will assume that $L$ fixes $S$ pointwise.

9. The cases $n=2,3$. We must handle $n=2,3$ separately.

Proposition 9.1. The main Theorem is valid when $n=2$.

Proof. Every member of $G$ is in one of two subspaces of $M(2, K)$

$$
\begin{aligned}
& V_{1}=\left\langle\left(\begin{array}{ll}
1 & 0 \\
0 & 1
\end{array}\right),\left(\begin{array}{rr}
0 & 1 \\
-1 & 0
\end{array}\right)\right\rangle \\
& V_{2}=\left\langle\left(\begin{array}{rr}
1 & 0 \\
0 & -1
\end{array}\right),\left(\begin{array}{ll}
0 & 1 \\
1 & 0
\end{array}\right)\right\rangle .
\end{aligned}
$$

Now $\left\langle\left(\begin{array}{rr}0 & 1 \\ -1 & 0\end{array}\right)\right\rangle$ is the tangent space and hence is invariant under $L$. Thus, $L\left(V_{1}\right)=V_{1}$ and hence $L\left(V_{2}\right)=V_{2}$. Since skews are mapped to skews, $L\left(\left(\begin{array}{rr}0 & 1 \\ -1 & 0\end{array}\right)\right)= \pm\left(\begin{array}{rr}0 & 1 \\ -1 & 0\end{array}\right)$. Conjugate with $\left(\begin{array}{rr}1 & 0 \\ 0 & -1\end{array}\right)$ if necessary to assume that $V_{1}$ is pointwise fixed. 
Let $L\left(\begin{array}{rr}1 & 0 \\ 0 & -1\end{array}\right)=\left(\begin{array}{rr}a & b \\ b & -a\end{array}\right)$. Since $\left(\begin{array}{rr}a & b \\ b & -a\end{array}\right)$ is a symmetry, it is $G$-conjugate to $\left(\begin{array}{rr}1 & 0 \\ 0 & -1\end{array}\right)$ and hence we may assume $\left(\begin{array}{rr}1 & 0 \\ 0 & -1\end{array}\right)$ is fixed and $V_{1}$ is still pointwise fixed, by taking the transpose if necessary.

Put $\quad L\left(\begin{array}{ll}0 & 1 \\ 1 & 0\end{array}\right)=\left(\begin{array}{cc}a & b \\ b & -a\end{array}\right)$. Then $\quad\left(\begin{array}{cc}a+1 & b \\ b & -a-1\end{array}\right) \in$ $\sqrt{2} G$. Thus, $a=0, b= \pm 1$. If $b=1$, we are done. If $b=-1$, conjugate with $\left(\begin{array}{rr}1 & 0 \\ 0 & -1\end{array}\right)$ and then apply the transpose map.

PROPOSITION 9.2. If $n=3$, the restriction of $L$ to the symmetric matrices is a $G$-conjugation.

Proof. By Proposition 8.6, every symmetry $\sigma$ is mapped to a symmetry. Let $\left\langle v_{1}\right\rangle$ be the line of reflection of $\sigma$ and let $v_{1}, v_{2}, v_{3}$ be an orthonormal basis of $V$. We may assume $L(\sigma)=\sigma$. Since $L$ preserves orthogonality, (Proposition 8.5 works when $n=3$ ) any symmetry reflecting in $\left\langle v_{2}, v_{3}\right\rangle$ is mapped to a symmetry reflecting in $\left\langle v_{2}, v_{3}\right\rangle$. Thus, from Proposition 9.1, we have that the space

$$
\left(\begin{array}{lll}
c & 0 & 0 \\
0 & a & b \\
0 & b & d
\end{array}\right)
$$

is acted upon by $L$ by $G$-conjugation. Hence, we assume this space is fixed pointwise.

Consider the image $B$ of the symmetry

$$
\left(\begin{array}{lll}
0 & 1 & 0 \\
1 & 0 & 0 \\
0 & 0 & 1
\end{array}\right)
$$

Since orthogonality of nonisotropic lines is preserved, $B$ commutes with $\operatorname{diag}(c, c,-c)$ for all $c$. Thus

$$
L(B)=\left(\begin{array}{lll}
0 & 1 & 0 \\
1 & 0 & 0 \\
0 & 0 & 1
\end{array}\right)
$$

A similar argument on

$$
\left(\begin{array}{lll}
0 & 0 & 1 \\
0 & 1 & 0 \\
1 & 0 & 0
\end{array}\right)
$$

finishes the proof. 
10. The tangent space. We have $\Omega=$ the tangent space of $G$, i.e., $\Omega$ is the space of skew-symmetric matrices. We need to show that $L \mid \Omega= \pm$ identity. For $i<j$, let $F_{i j}$ be the $n \times n$ matrix whose $(i, j)$ entry is 1 , whose $(j, i)$ entry is -1 , and all other entries are 0 .

Proposition 10.1. If for all $i<j, L\left(F_{i j}\right)= \pm F_{i j}$, then $L / \Omega$ is \pm identity.

Proof. Use induction on $n$. We are done if $n=2$. Let $A_{1} \in M(n-1, K), \quad A_{1} \quad$ skew. Then $0 \dot{+} A_{1} \rightarrow 0 \dot{+} B_{1}, \quad B_{1} \in$ $M(n-1, K)$. By induction, $B_{1}=A_{1}$ for all skew $A_{1}$. Suppose $L\left(F_{12}\right)=-F_{12}$. Note that

$$
\left[\begin{array}{ccc}
0 & \frac{1}{\sqrt{2}} & \frac{1}{\sqrt{2}} \\
-\frac{1}{\sqrt{2}} & \frac{1}{2} & -\frac{1}{2} \\
\frac{1}{\sqrt{2}} & \frac{1}{2} & -\frac{1}{2}
\end{array}\right] \in G .
$$

However, all symmetric matrices are fixed, and hence the above matrix is mapped to

$$
\left[\begin{array}{ccc}
0 & -\frac{1}{\sqrt{2}} & \frac{1}{\sqrt{2}} \\
\frac{1}{\sqrt{2}} & \frac{1}{2} & -\frac{1}{2} \\
\frac{1}{\sqrt{2}} & \frac{1}{2} & -\frac{1}{2}
\end{array}\right] \notin G .
$$

The above argument may be repeated for all $F_{i j}$.

Proposition 10.2. For all $i<j, L\left(F_{i j}\right)= \pm F_{i j}$.

Proof. Let $A=\operatorname{diag}\left(\epsilon_{1}, \cdots, \epsilon_{i-1}, 0, \epsilon_{i+1}, \cdots, \epsilon_{j-1}, 0, \epsilon_{j+1}, \cdots, \epsilon_{n}\right)$ where the $\epsilon_{k}$ run through \pm 1 . Put $B=L\left(F_{i j}\right)$. Now $A \pm B \in G$. Thus,

$$
\begin{aligned}
& A A^{\prime}+B B^{\prime}=I_{n} \\
& A B^{\prime}+B A^{\prime}=0 .
\end{aligned}
$$

It follows that $B$ has the appropriate form and the proposition is proved. This also concludes the proof of the main Theorem. 
We would like to thank the referee and Professor D. Shapiro for many suggestions and corrections.

\section{REFERENCES}

1. E. Artin, Geometric Algebra, Interscience, 1957.

2. C. Chevalley, Théorie des Groupes de Lie, Hermann, Paris, 1968.

3. J. Dieudonné, The Automorphisms of the classical groups, Amer. Math. Soc. Memoirs, 1951.

4. - Sur une généralisation du groupe orthogonal à quatre variables, Arch. Math., 1 (1949), 282-287.

5. M. Marcus, All linear operators leaving the unitary group invariant, Duke Math. J., 26 (1959), 155-163.

6. L Linear transformations on matrices, J. Res. Nat. Bur. Standards, 75B, No. 3, 107-114.

7. M. Marcus and R. Purves, Linear transformations on algebras of matrices II, Canad. J. Math., 11 (1959), 383-396.

8. S. Pierce, Linear operators preserving the real symplectic group, Canad. J. Math., (1975), 715-724.

9. W. E. Roth, On direct product matrices, Bull. Amer. Math. Soc., 40 (1934), 461-468.

10. A. Wei, Linear operators preserving the real orthogonal group, Canad. J. Math., (1975), 561-572.

Received March 23, 1976. The work of the second author was partially supported by NRC Grant A-7862.

UNIVERSITY OF TORONTO

Toronto, Canada M5S 1A1 




\section{Pacific Journal of Mathematics \\ Vol. 70, No. $1 \quad$ September, 1977}

William H. Barker, Noether's theorem for plane domains with hyperelliptic

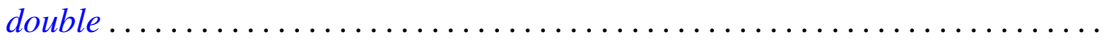

Michael James Beeson, Non-continuous dependence of surfaces of least area on the boundary curve ...................................... 11

Horst Behncke, Functions acting in weighted Orlicz algebras . . . . . . . . . . . . 19

Howard Edwin Bell, A commutativity study for periodic rings . . . . . . . . . . . 29

Peter Botta and Stephen J. Pierce, The preservers of any orthogonal group ....... 37

Douglas S. Bridges, The constructive Radon-Nikodým theorem ............. 51

James Dennis Brom, The theory of almost periodic functions in constructive

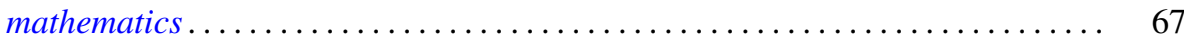

N. Burgoyne and C. Williamson, Semi-simple classes in Chevalley type groups ....

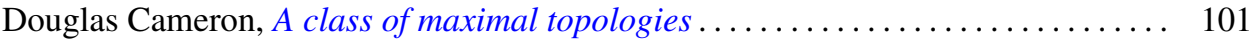

L. Carlitz, Enumeration of doubly up-down permutations . . . . . . . . . . . . . . 105

Paul Robert Chernoff, The quantum n-body problem and a theorem of

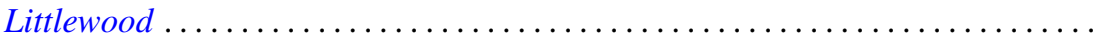

Jo-Ann Deborah Cohen, Locally bounded topologies on $F(X) \ldots \ldots \ldots \ldots \ldots \ldots$

Heinz Otto Cordes and Robert Colman McOwen, Remarks on singular elliptic

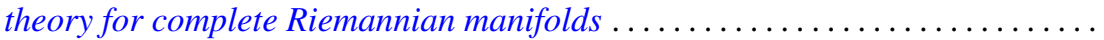

Micheal Neal Dyer, Correction to: "Rational homology and Whitehead

products"

Robert Fernholz, Factorization of Radonifying transformations

Lawrence Arthur Fialkow, A note on quasisimilarity. II ...... . .

Harvey Charles Greenwald, Lipschitz spaces of distributions on the surface of unit

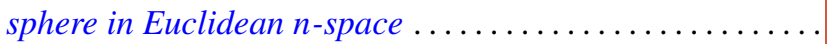

Albrecht Irle, On the measurability of conditional expectations

Tom (Roy Thomas Jr.) Jacob, Matrix transformations involving simple sequence

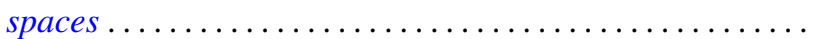

A. Katsaras, Continuous linear maps positive on increasing continuous

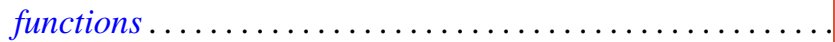

Kenneth Kunen and Judith Roitman, Attaining the spread at cardinals of cofinality

Lawrence Louis Larmore and Robert David Rigdon, Enumerating normal bundles

of immersions and embeddings of projective spaces ...... . .

Ch. G. Philos and V. A. Staïkos, Asymptotic properties of nonoscillatory solutions of differential equations with deviating argument .

Peter Michael Rosenthal and Ahmed Ramzy Sourour, On operator algebras containing cyclic Boolean algebras...

Polychronis Strantzalos, Strikt fast gleichgradig-stetige und eigentliche

Aktionen ...

Glenn Francis Webb, Exponential representation of solutions to an abstract

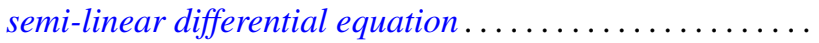

